\title{
Low cardiac output as physiological phenomenon in hibernating, free-ranging Scandinavian brown bears (Ursus arctos) - an observational study
}

\author{
Peter Godsk Jørgensen ${ }^{1 *}$, Jon Arnemo ${ }^{2,3}$, Jon E Swenson ${ }^{4,5}$, Jan S Jensen ${ }^{1}$, Søren Galatius ${ }^{1}$ and Ole Frøbert ${ }^{6}$
}

\begin{abstract}
Background: Despite 5-7 months of physical inactivity during hibernation, brown bears (Ursus arctos) are able to cope with physiological conditions that would be detrimental to humans. During hibernation, the tissue metabolic demands fall to $25 \%$ of the active state. Our objective was to assess cardiac function associated with metabolic depression in the hibernating vs. active states in free-ranging Scandinavian brown bears.

Methods: We performed echocardiography on seven free-ranging brown bears in Dalarna, Sweden, anesthetized with medetomidine-zolazepam-tiletamine-ketamine during winter hibernation in February 2013 and with medetomidine-zolazepam-tiletamine during active state in June 2013. We measured cardiac output noninvasively using estimates of hemodynamics obtained by pulsed wave Doppler echocardiography and 2D imaging. Comparisons were made using paired T-tests.

Results: During hibernation, all hemodynamic indices were significantly decreased (hibernating vs. active state): mean heart rate was 26.0 (standard deviation (SD): 5.6) beats per min vs. 75.0 (SD: 17.1) per min ( $P=0.002$ ), mean stroke volume 32.3 (SD: 5.2) $\mathrm{ml}$ vs. 47.1 (SD: 7.9) $\mathrm{ml}(\mathrm{P}=0.008$ ), mean cardiac output 0.86 (SD: 0.31) l/min vs. 3.54 (SD: 1.04) $\mathrm{I} / \mathrm{min}\left(P=0.003\right.$ ), and mean cardiac index 0.63 (SD: 0.21) l/min/kg vs. 2.45 (SD: 0.52) I/min/ $\mathrm{m}^{2}(P<0.001)$. Spontaneous echo contrast was present in all cardiac chambers in all seven bears during hibernation, despite the absence of atrial arrhythmias and valvular disease.

Conclusion: Free-ranging brown bears demonstrate hemodynamics comparable to humans during active state, whereas during hibernation, we documented extremely low-flow hemodynamics. Understanding these physiological changes in bears may help to gain insight into the mechanisms of cardiogenic shock and heart failure in humans.
\end{abstract}

Keywords: Animal model cardiovascular disease, Acute cardiac care, Thrombosis, Echocardiography

\section{Background}

Brown bears (Ursus arctos) have unique physiological adaptations to deal with the annual hibernation period lasting 5-7 months, in which they do not eat, drink, defecate, or urinate and display minimal physical activity [1]. These adaptations protect the bears from the detrimental effects of inactivity and, hence, they avoid the loss of muscle and bone mass [2-5], decubitus ulcers, and the deterioration of cardiac function [6-9]. During this period the bears' oxygen demand is reduced to $25 \%$

\footnotetext{
*Correspondence: petergodsk@gmail.com

'Department of Cardiology, University of Copenhagen, Gentofte Hospital, Copenhagen, Denmark

Full list of author information is available at the end of the article
}

of the active state [10] and cardiac adaptations, including profound bradycardia and low cardiac output are induced to optimize energy conservation during the long period of low metabolism. Whereas bed rest and sitting still for longer periods of time predispose humans to thromboembolism [11], this is apparently not the case in the brown bear. Clearly, the identification of mechanisms responsible for these adaptations could have substantial applications for various areas of human medicine including intensive care medicine and in prevention of thromboembolism.

In humans, spontaneous echo contrast (SEC) in the echocardiogram is the presence of swirling echo-dense shadows in the cardiac chambers and large vessels. SEC occurs in areas of low blood flow, and is caused by the 
aggregation of red blood cells and plasma proteins $[12,13]$. The presence of SEC is strongly associated with cardiac pathophysiology in humans and predisposes to thromboembolic events [13-18].

Reduced cardiac output during hibernation has been described in bears previously $[7,8]$ but a more detailed description of the cardiac functional adaptations is warranted for a deeper understanding of the physiology of the brown bears. In addition, the previous studies were conducted in captive bears with the risk of introducing bias caused by human interaction and/or presence during hibernation [19]. Hence, the aim of this study was to provide a detailed documentation of the cardiac function and intracardiac blood flow pattern in the hibernating and in the active free-ranging brown bears.

\section{Methods}

\section{Material}

We assessed the hemodynamics of seven subadult hibernating brown bears in Dalarna, Sweden in February 2013. These bears had received GPS collars previously, which allowed us to locate them in their dens. The bears were immobilized using a mixture of medetomidine, zolazepam, tiletamine and ketamine [20]. The same bears, in the active state, were immobilized in June 2013, where they were darted from a helicopter with medetomidine, zolazepam and tiletamine at 2-4 times the winter dose [20]. The study was approved by the Swedish Ethical Committee on Animal Research (C212/9) and the procedure was in compliance with Swedish laws and regulations.

\section{Echocardiography}

We performed echocardiography in the field with the bear in a left lateral recumbency using a Phillips CX50 with an S51 probe. We used second harmonic imaging and the obtained images included 2D, MMode, and pulsed and continuous wave spectral Doppler echocardiography. We estimated stroke volume using the formula.

$$
\mathrm{SV}=\pi *(\mathrm{D} / 2)^{2} \times \mathrm{TVI}
$$

where SV is the stroke volume $(\mathrm{ml}), \mathrm{D}$ is the left ventricular outflow tract (LVOT) diameter $(\mathrm{cm})$, measured in the parasternal long axis view, and TVI is the time velocity integral $(\mathrm{cm})$ of the pulsed wave spectral Doppler in the LVOT after careful alignment of the marker in the direction of the blood flow. To minimize the influence of measurement error of the LVOT diameter, we used each bear's mean value of LVOT diameter measured during hibernation and active state to calculate SV. Cardiac output (l/min) was calculated using the formula.

$$
\mathrm{CO}=\mathrm{SV} \times \mathrm{HR} \times 1000
$$

where HR (beats/s) is the mean heart rate obtained from 6 heart beats. We calculated the cardiac index (CI) $\left(\mathrm{l} / \mathrm{min} / \mathrm{m}^{2}\right)$ using an estimation of body surface area (BSA) $\left(\mathrm{m}^{2}\right)$ as previously described [21].

$$
\mathrm{CI}=\mathrm{CO} / \mathrm{BSA}
$$

We measured ejection time and time to peak velocity from the pulsed wave spectral Doppler placed in LVOT as above. We obtained cardiac intervals with the pulsed wave spectral Doppler placed between mitral leaflets during diastole. Isovolumetric relaxation time (IVRT) + Isovolumetric contraction time (IVCT) was calculated as end of A wave to start of $\mathrm{E}$ wave minus ejection time and Tei index was calculated as (IVRT + IVCT)/ejection time.

Echocardiograms, post-processing, and assessment of SEC were performed by an experienced echocardiographer (PGJ).

\section{Statistics}

The values are presented as mean \pm 1 standard deviation (SD). Because of the small number of bears, normal distribution of values was difficult to assume. However, the results of the paired T-tests were chosen to be shown with the limitation that normal distribution of data might not be fulfilled. The calculations were made using the statistical software package ' $R$ ', version 3.0.1 (64 bit) (R Project for Statistical Computing, http://www.R-project.org).

\section{Results and discussion}

In total, we examined the same seven bears in the field in both February and June. Echocardiograms were only obtained in six of the bears ( 4 females and 2 males) in June 2013. During hibernation the bears did not differ significantly from the active state in body mass $(50.3 \mathrm{~kg}$ vs. $54.3 \mathrm{~kg}$; $\mathrm{P}=0.50$ ).

We found no valve disorders or arrhythmias, during the echocardiographic examinations.

Flow indices for each bear and mean values are found in Figure 1. During hibernation, all flow indices were reduced compared with the active state (hibernating vs. active state): heart rate was reduced to $35 \%(26.0( \pm 5.6)$ beats per min vs. $75.0( \pm 17.1)$ beats per $\min (P=0.002))$; stroke volume to $69 \%(32.3( \pm 5.2) \mathrm{ml}$ vs. $47.1( \pm 7.9) \mathrm{ml}$ $(\mathrm{P}=0.008))$; cardiac output to $24 \%(0.86( \pm 0.31) \mathrm{l} / \mathrm{min}$ vs. $3.54( \pm 1.04) \mathrm{l} / \mathrm{min}(\mathrm{P}=0.003))$ and cardiac index to $26 \%\left(0.63( \pm 0.21) \mathrm{l} / \mathrm{min} / \mathrm{m}^{2}\right.$ vs. $2.45( \pm 0.52) \mathrm{l} / \mathrm{min} / \mathrm{m}^{2}$ $(\mathrm{P}<0.001))$.

Pulsed wave Doppler-derived measurements of cardiac intervals are found in Table 1. Both systolic and diastolic time intervals were increased during hibernation. However, although ejection time was increased by $30 \%$ and time to peak velocity was increased by $70 \%$, the most prominent increase was in diastolic filling 


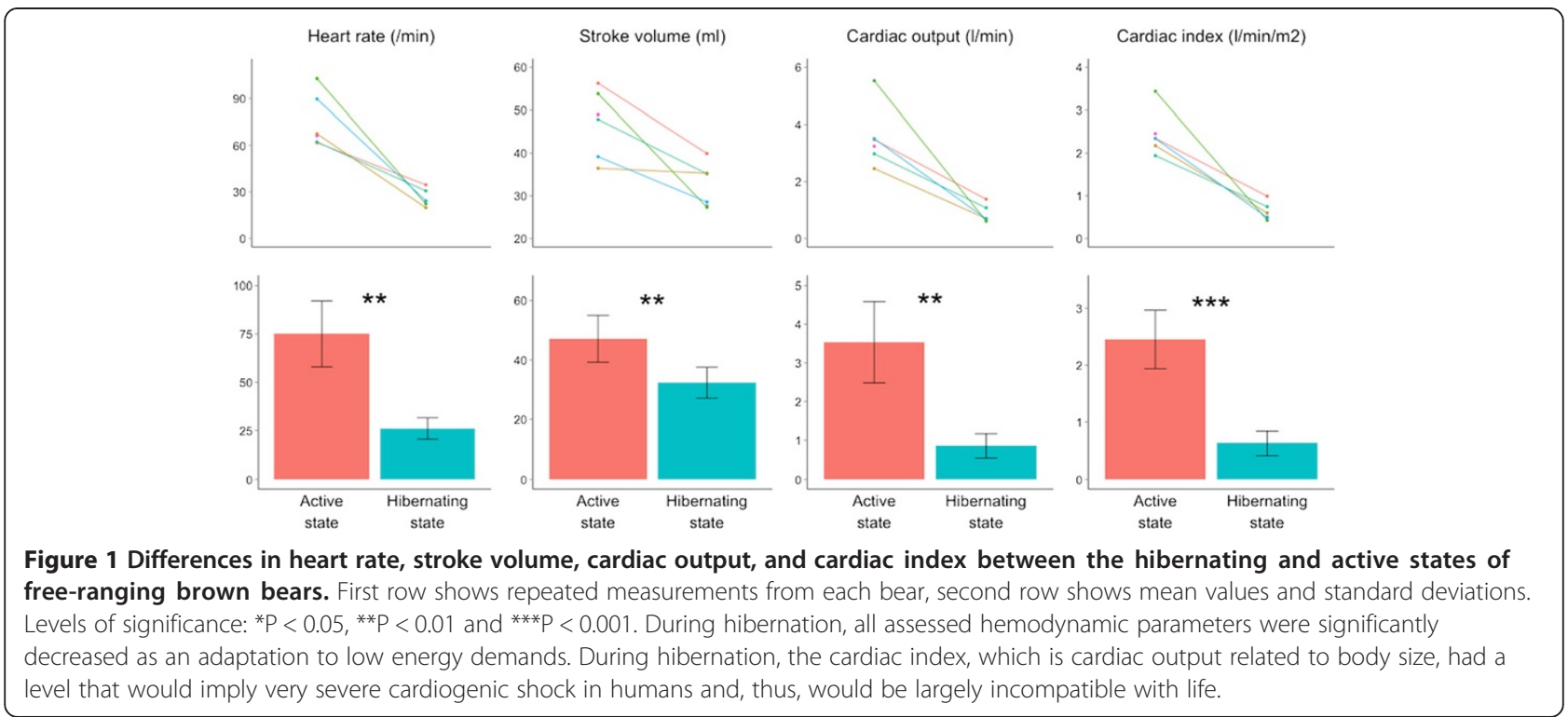

time, which increased by a factor 3.9. Likewise, the Tei index was increased by $66 \%$ during hibernation, indicating relatively longer periods of isovolumetric phases.

SEC was present as swirling echo-dense structures in all four chambers of all seven bears examined in the hibernating state in February 2013. However, there were no signs of SEC in the bears when examined during their active state in June 2013 (Figure 2 and Additional files 1 and 2).

In this study, we describe the pattern of extreme low flow hemodynamics in hibernating brown bears. Although all measured flow indices were significantly reduced during hibernation, the difference was most pronounced for $\mathrm{CO}$, with more than a four-fold decrease during hibernation. In addition, even $\mathrm{CI}$, which takes in to account both differences in HR, SV and animal size and provides the most accurate assessment of changes in hemodynamics, was increased almost four-fold during the active state. The low flow hemodynamics were reflected by the presence of SEC in all cardiac chambers.

Table 1 Cardiac time intervals measured by pulsed wave doppler echocardiography

\begin{tabular}{lccc}
\hline & Active state & Hibernating state & P-value \\
\hline Ejection time $(\mathrm{ms})$ & $200( \pm 18.6)$ & $256( \pm 16.9)$ & 0.005 \\
Diastolic filling time $(\mathrm{ms})$ & $571( \pm 103)$ & $2217( \pm 858)$ & 0.02 \\
IVRT + IVCT (ms) & $363( \pm 30)$ & $583( \pm 61)$ & 0.006 \\
Tei index & $0.77( \pm 0.10)$ & $1.28( \pm 0.29)$ & 0.07 \\
\hline
\end{tabular}

IVRT = isovolumetric ventricular relaxation time, IVCT = isovolumetric ventricular contraction time.
Low blood flow hemodynamics and metabolism: potential perspectives for human medicine

All assessed hemodynamic parameters (SV, CO, CI) were significantly reduced during hibernation, indicating adaptation to low energy demands. The magnitude of this metabolic change can be illustrated by comparing to human values, where $\mathrm{CI}$ is considered normal when between 2.6 and $4.2 \mathrm{l} / \mathrm{min} / \mathrm{m}^{2}$ and cardiogenic shock may be present when the $\mathrm{CI}$ drops below $1.8 \mathrm{l} / \mathrm{min} / \mathrm{m}^{2}$ [22]. Thus, the CI during the bears' active state was within the normal range for humans, but during hibernation it was reduced to an extent that is largely incompatible with life in humans. Whereas treatment of cardiogenic shock is traditionally centered on reestablishing adequate tissue perfusion by improving cardiac output [22], there is less focus on minimizing tissue damage by decreasing the metabolic demands in the peripheral tissues.

In some clinical settings, such as after cardiac arrest and during cardiac surgery, induction of slight hypothermia is an established treatment. In these cases, hypothermia acts by suppressing metabolism and is thought to prevent primarily brain damage during and after periods of impaired end-organ oxygen delivery [23]. However, according to a recent study that examined the cardiac effects of therapeutic hypothermia as measured with echocardiography; [24] the isolated hypothermic effect in pigs is different from the adaptations in the hypothermic, hibernating bear. In hypothermic pigs like in hibernating bears, the cardiac output was slightly decreased and the duration of the systole was increased with increased ejection time. In hypothermic pigs, however, heart rate and duration of the diastole were slightly reduced while, in the hibernating bear, heart rate was about one third of the active state 


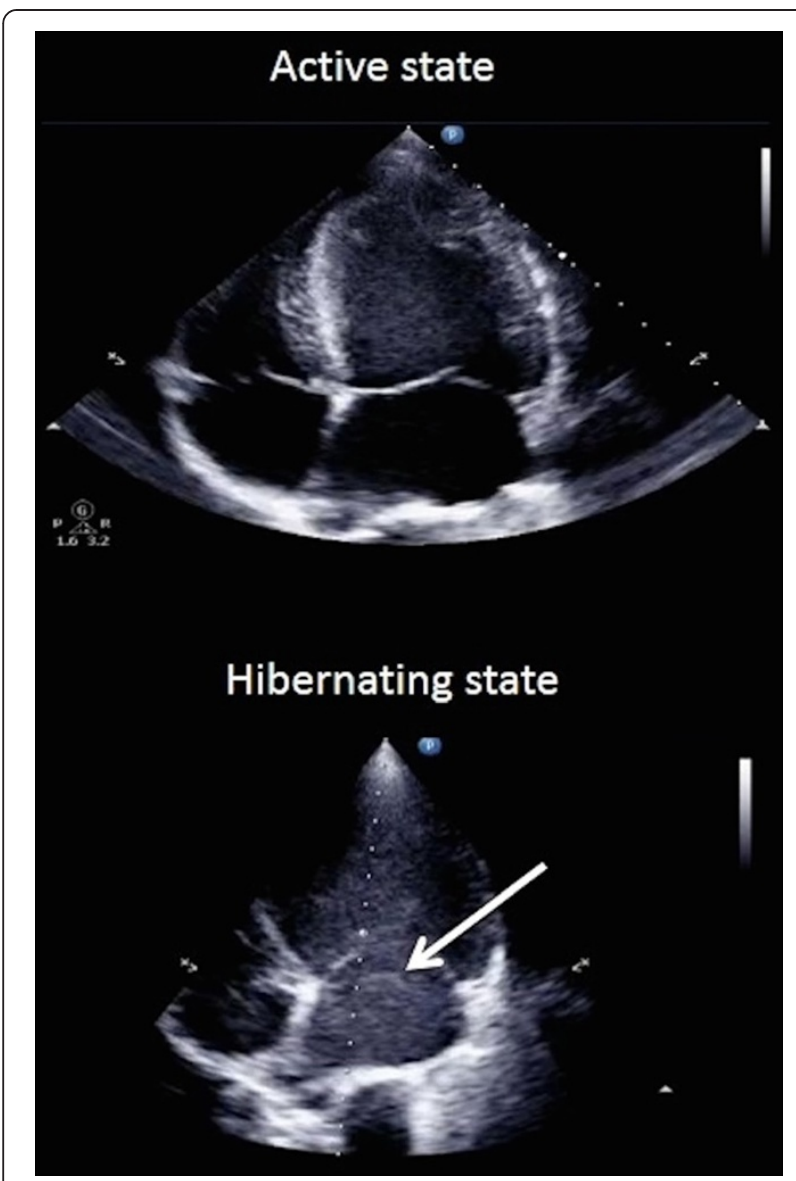

Figure 2 Comparison of 2D echocardiographic images from the hibernating and active states of free-ranging brown bears. An arrow indicates the presence of spontaneous echo contrast as echo-dense shadows during hibernation. Also see Additional files 1 and 2.

and diastolic filling time increased almost 4 fold. Hence, our data suggest that the cardiac adaptations evident during hibernation are different from that obtained by mere hypothermic metabolic suppression. This is supported by a recent study, showing that metabolic suppression in hibernating American black bears (Ursus americanus) is independent of lowered body temperature [10].

A substance derived from the serum of hibernators with the ability to induce a hibernation-like state in nonhibernators may exist $[25,26]$. This so-called 'hibernation-inducing trigger' has also been identified in hibernating bear serum, and there is evidence that exposing rabbits' hearts to hibernating bear serum prior to cardioplegia reduces ischemia-reperfusion injury [27], thus indicating that bear serum has the capability to reduce myocardial oxygen demands similar to the hibernating myocardium. Other substances that can induce hibernation-like states in nonhibernating animals also exist. Exposing mice to hydrogen sulfide $\left(\mathrm{H}_{2} \mathrm{~S}\right)$ induces a 'suspended animation-like state', with decreased metabolism, core body temperature [28], and cardiac and respiratory effects very similar to those found in the hibernating bear [29].

\section{Spontaneous echo contrast - evidence of cause and consequences}

Blood is usually echo-lucent in the cardiac chambers. Occasionally blood appears as a swirling haze of echodense structures, which is caused by the aggregation of red blood cells and plasma proteins $[12,13]$ and is exclusively seen under conditions of low blood flow, or stasis, with low shear stress. Dense SEC has even been suggested to be a transition state in the formation of fibrinrich, red thrombi [30,31]. In humans, conditions predisposing to low flow and/or stasis conditions include atrial fibrillation, mitral stenosis, and dilated cardiomyopathy. In all three conditions, the presence of SEC is an ominous sign of risk for future thromboembolic disease and is used to guide clinical decision-making on a daily basis [13-18]. Though the mechanisms of SEC may be species specific (making it difficult to directly compare the species), our findings suggest that the mechanisms underlying the formation of SEC persist and remain unaltered during the brown bears' hibernation period. This, in turn, implies that the adaptations in blood coagulation during hibernation might involve hematological mechanisms not essential for SEC formation.

\section{Clinical perspectives}

The hibernating bear model reminds the clinicians that metabolism is not a fixed entity, but that it is perpetually adjusting to the prevailing circumstances. Careful adjustment of treatment is advised when using medications that modify metabolism in critically ill patients. This is, for instance, the case with vasoactive agents, like catecholeamines, that in addition to their vasoactive effect, increase organ metabolism, and hence oxygen consumption [32]. The existence of substances that can induce a hibernation-like state in nonhibernating animals indicates that low-metabolism conditions can be medically induced in other species and opens for more research on possible applications for humans.

The finding of SEC in all cardiac chambers in the hibernating bear, which is apparently free from thromboembolic events, suggests that the increased risk of thromboembolic events in humans with SEC is not consequence of SEC itself, but may merely be a marker of the severity of the underlying disease.

\section{Strengths and limitations}

The free-ranging brown bear used in the present study is a strong model to understand biomimicry, the imitation of models in nature for the purpose of solving complex human problems, because bears are undisturbed by human interaction and/or presence. This allows for the 
study of bears in their natural habitat, without potential bias introduced by captivity, such as audible, visual, and olfactory stimuli and unnatural food availability. Our model, however, is limited by the fact that the bears were immobilized using anesthetics, which might induce changes in hemodynamic parameters [8]. Our finding of reduced SV differs from previous studies on unanesthetized bears and thus the measurements might have been affected by the anesthesia, differences in methods used to calculate SV, or a combination of both. However, we consider this unlikely, because of the lower doses of anesthetics used in winter compared with summer captures. Moreover, the differences we found in $\mathrm{HR}, \mathrm{CO}$, and CI agree with earlier studies [8]. SEC was only present in the hibernating state and with reference to doses. We find no reason to believe that the described low-flow conditions and the presence of SEC were caused solely by the use of anesthetics.

A more comprehensive echocardiographic examination would have been preferable. However, taken into account the circumstances of especially the winter examinations the sub-zero temperatures, limited time-frame for the echocardiographic examination and the need to limit the area that is shaved on the bear - the number of measurements to be collected in a reproducible manner is limited.

\section{Conclusion}

Low blood flow hemodynamics and SEC are normal physiological phenomena in free-ranging Scandinavian brown bears in response to low energy demands during hibernation. Identification of the mechanisms responsible for the lowered metabolic rate and decreased hemodynamic indices could have implications for human medicine, including treatment of cardiogenic shock, heart failure and prevention of thromboembolism.

\section{Additional files}

Additional file 1: 2D echocardiographic images from the hibernating state of free-ranging brown bears. The presence of spontaneous echo contrast as swirling, echo-dense shadows is easily recognized in the left atrium and ventricle. The blood is almost entirely halted during parts of the prolonged diastole.

Additional file 2: 2D echocardiographic images from the active state of free-ranging brown bears. There is no evidence of spontaneous echo contrast in neither left atrium nor ventricle.

\section{Abbreviations}

BSA: Body surface area; Cl: Cardiac index; CO: Cardiac output; HR: Heart rate; IVCT: Isovolumetric contraction time; IVRT: Isovolumetric relaxation time; LVOT: Left ventricular outflow tract; SD: Standard deviation; SEC: Spontaneous echo contrast; SV: Stroke volume; TVI: Time velocity integral.

\section{Competing interest}

The authors declare that they have no competing interests.

\section{Authors' contributions}

PGJ participated in the design of the study, acquisition and analysis of data and drafted the manuscript. JA participated in the design and the acquisition of data. JES participated in the design. JSJ assisted in the analysis and interpretation of data. SG participated in the design and the interpretation of data. OF participated in the design and the acquisition and analysis of the data. All authors read and approved the final manuscript.

\section{Acknowledgements and funding}

We would like to thank Lisbeth Høier Olsen, Professor, DVM, DVSc, Department of Veterinary Disease Biology University of Copenhagen and Mette Flethøj Madsen, DVM, PhD student, University of Copenhagen for invaluable echocardiographic support prior to the field study and Sven Brunberg for excellent organisation of the field work.

The Scandinavian Brown Bear Research Project was funded by the Swedish Environmental Protection Agency, the Norwegian Directorate for Nature Management, the Swedish Association for Hunting and Wildlife Management, the Research Council of Norway, the Austrian Science Fund, a Nordforsk researcher network grant (project no. 44042), and the Lundbeck Foundation (R126-2012-12408). This is paper number 174 from the Scandinavian Brown Bear Research Project.

\section{Author details}

${ }^{1}$ Department of Cardiology, University of Copenhagen, Gentofte Hospital, Copenhagen, Denmark. ${ }^{2}$ Department of Forestry and Wildlife Management, Faculty of Applied Ecology and Agricultural Sciences, Hedmark College, Campus Evenstad, NO-2418 Elverum, Norway. ${ }^{3}$ Department of Wildlife, Fish and Environmental Studies, Faculty of Forest Sciences, Swedish University of Agricultural Sciences, SE-901 83 Umeå, Sweden. ${ }^{4}$ Department of Ecology and Natural Resource Management, Norwegian University of Life Sciences, NO-1528 Ås, Norway. ${ }^{5}$ Norwegian Institute for Nature Research, NO-7485 Trondheim, Norway. 'Department of Cardiology, Örebro University Hospital, Örebro, Sweden.

Received: 15 August 2014 Accepted: 9 September 2014

Published: 16 September 2014

\section{References}

1. Manchi S, Swenson J: Denning behaviour of Scandinavian brown bears Ursus arctos. Wildl Biol 2005, 11:125-132.

2. Shavlakadze T, Grounds M: Of bears, frogs, meat, mice and men: complexity of factors affecting skeletal muscle mass and fat. BioEssays 2006, 28:994-1009.

3. Donahue SW, Galley SA, Vaughan MR, Patterson-Buckendahl P, Demers LM, Vance JL, McGee ME: Parathyroid hormone may maintain bone formation in hibernating black bears (Ursus americanus) to prevent disuse osteoporosis. J Exp Biol 2006, 209:1630-1638.

4. Vestergaard P, Støen O-G, Swenson JE, Mosekilde L, Heickendorff L, Fröbert O: Vitamin D status and bone and connective tissue turnover in brown bears (Ursus arctos) during hibernation and the active state. PLOS One 2011, 6:e21483.

5. Fink T, Rasmussen JG, Emmersen J, Pilgaard L, Fahlman Å, Brunberg S, Josefsson J, Arnemo JM, Zachar V, Swenson JE, Fröbert O: Adipose-derived stem cells from the brown bear (Ursus arctos) spontaneously undergo chondrogenic and osteogenic differentiation in vitro. Stem Cell Res 2011, 7:89-95.

6. Nelson OL, McEwen M-M, Robbins CT, Felicetti L, Christensen WF: Evaluation of cardiac function in active and hibernating grizzly bears. $J$ Am Vet Med Assoc 2003, 223:1170-1175.

7. Nelson OL, Robbins CT, Wu Y, Granzier H: Titin isoform switching is a major cardiac adaptive response in hibernating grizzly bears. Am J Physiol - Heart Circ Physiol 2008, 295:H366-H371.

8. Nelson OL, Robbins CT: Cardiac function adaptations in hibernating grizzly bears (Ursus arctos horribilis). J Comp Physiol B 2010, 180:465-473.

9. Laske TG, Harlow HJ, Garshelis DL, laizzo PA: Extreme respiratory sinus arrhythmia enables overwintering black bear survival-physiological insights and applications to human medicine. J Cardiovasc Trans/ Res 2010, 3:559-569.

10. Tøien $\varnothing$, Blake J, Edgar DM, Grahn DA, Heller HC, Barnes BM: Hibernation in black bears: independence of metabolic suppression from body temperature. Science 2011, 331:906-909. 
11. Aldington S, Pritchard A, Perrin $K$, James $K$, Wijesinghe $M$, Beasley R: Prolonged seated immobility at work is a common risk factor for venous thromboembolism leading to hospital admission. Intern Med J 2008, 38:133-135.

12. Merino A, Hauptman P, Badimon L, Badimon JJ, Cohen M, Fuster V, Goldman M: Echocardiographic "smoke" is produced by an interaction of erythrocytes and plasma proteins modulated by shear forces. J Am Coll Cardiol 1992, 20:1661-1668

13. Black IW, Chesterman CN, Hopkins AP, Lee LCL, Chong BH, Walsh WF: Hematologic correlates of left atrial spontaneous echo contrast and thromboembolism in nonvalvular atrial fibrillation. J Am Coll Cardio/ 1993, 21:451-457.

14. Black IW, Hopkins AP, Lee LCL, Walsh WF: Left atrial spontaneous echo contrast: a clinical and echocardiographic analysis. J Am Coll Cardiol 1991, 18:398-404.

15. Leung DYC, Black IW, Cranney GB, Hopkins AP, Walsh WF: Prognostic implications of left artial spontaneous echo contrast in nonvalvular atrial fibrillation. J Am Coll Cardiol 1994, 24:755-762.

16. Iliceto S, Antonelli G, Sorino M, Biasco G, Rizzon P: Dynamic intracavitary left atrial echoes in mitral stenosis. Am J Cardiol 1985, 55:603-606.

17. Kim M-K, Park D-G: Correlation between stroke and spontaneous echo contrast by tissue harmonic imaging in patients with dilated cardiomyopathy. J Cardiovasc Ultrasound 2009, 17:10.

18. Shen WF, Tribouilloy C, Rida Z, Peltier M, Choquet D, Rey JL, Lesbre JP: Clinical significance of intracavitary spontaneous echo contrast in patients with dilated cardiomyopathy. Cardiology 1996, 87:141-146.

19. Hissa R: Physiology of the European brown bear (Ursus arctos arctos). Ann Zool Fenn 1997, 34:267-287.

20. Evans AL, Sahlén V, Støen O-G, Fahlman Å, Brunberg S, Madslien K, Fröbert $\mathrm{O}$, Swenson JE, Arnemo JM: Capture, anesthesia, and disturbance of free-ranging brown bears (Ursus arctos) during hibernation. PLOS One 2012, 7:e40520.

21. Hill RC, Scott KC: Energy requirements and body surface area of cats and dogs. J Am Vet Med Assoc 2004, 225:689-694.

22. Reynolds HR, Hochman JS: Cardiogenic shock current concepts and improving outcomes. Circulation 2008, 117:686-697.

23. Campos J-M, Paniagua P: Hypothermia during cardiac surgery. Best Pract Res Clin Anaesthesiol 2008, 22:695-709.

24. Espinoza A, Kerans V, Opdahl A, Skulstad H, Halvorsen PS, Bugge JF, Fosse E, Edvardsen T: Effects of therapeutic hypothermia on left ventricular function assessed by ultrasound imaging. J Am Soc Echocardiogr 2013, 26:1353-1363.

25. Dawe AR, Spurrier WA: Hibernation induced in ground squirrels by blood transfusion. Science 1969, 163:298-299.

26. Swan $\mathrm{H}$, Schätte $\mathrm{C}$ : Antimetabolic extract from the brain of the hibernating ground squirrel citellus tridecemlineatus. Science 1977, 195:84-85.

27. Bolling SF, Benedict MB, Tramontini NL, Kilgore KS, Harlow HH, Su T-P, Oeltgen PR: Hibernation triggers and myocardial protection. Circulation 1998, 98(19 Suppl):II220-II223.

28. Blackstone $\mathrm{E}: \mathrm{H} 2 \mathrm{~S}$ induces a suspended animation-like state in mice. Science 2005, 308:518-518.

29. Volpato GPMD, Searles RBA, Yu BPD, Scherrer-Crosbie MMD, Bloch KDMD, Ichinose FMD, Zapol WMMD: Inhaled hydrogen sulfide: a rapidly reversible inhibitor of cardiac and metabolic function in the mouse. Anesthesiol 2008, 108:659-668.

30. Beppu S, Nimura Y, Sakakibara H, Nagata S, Park Y-D, Izumi S: Smoke-like echo in the left atrial cavity in mitral valve disease: its features and significance. J Am Coll Cardiol 1985, 6:744-749.
31. Mikell FL, Asinger RW, Elsperger KJ, Anderson WR, Hodges M: Regional stasis of blood in the dysfunctional left ventricle: echocardiographic detection and differentiation from early thrombosis. Circulation 1982, 66:755-763.

32. Barth E, Albuszies G, Baumgart K, Matejovic M, Wachter U, Vogt J, Radermacher P, Calzia E: Glucose metabolism and catecholamines. Crit Care Med 2007, 35(9 Suppl):S508-S518.

doi:10.1186/1476-7120-12-36

Cite this article as: Jørgensen et al:: Low cardiac output as physiological phenomenon in hibernating, free-ranging Scandinavian brown bears (Ursus arctos) - an observational study. Cardiovascular Ultrasound 2014 12:36.

\section{Submit your next manuscript to BioMed Central and take full advantage of:}

- Convenient online submission

- Thorough peer review

- No space constraints or color figure charges

- Immediate publication on acceptance

- Inclusion in PubMed, CAS, Scopus and Google Scholar

- Research which is freely available for redistribution

Submit your manuscript at www.biomedcentral.com/submit
C) Biomed Central 